\title{
Determination of Surface Temperature in ICP RF Plasma Treatments of Organic Materials
}

\author{
Carlos Eduardo Farias ${ }^{a}$, Euclides Alexandre Bernardelli ${ }^{a}$, Paulo Cesar Borges ${ }^{a}$, Márcio Mafra ${ }^{a *}$ \\ ${ }^{a}$ Universidade Tecnológica Federal do Paraná - UTFPR, Curitiba, PR, Brazil
}

Received: March 01, 2017; Revised: May 30, 2017; Accepted: July 12, 2017

\begin{abstract}
The reactions that can occur on organic materials treated in plasma enviorement are strongly affected by surface temperature, thus the sharp determination of this variable is extremely important to process control. In situ temperature measurements are often employed; however, the measured point is generally under the treated surface. If in metallic materials the high thermal conductivity minimizes this problem, in non-metallic materials processing it becomes important, because its thermal resistivity besides a high sensibility to small temperature variations. The present work uses a simulation tool to extract thermal data on $\mathrm{Ar}+\mathrm{O}_{2} \mathrm{RF}$ plasma processing of Stearic Acid. Experimental data were obtained by thermocouples placed inside the samples. The extrapolation of surface temperature was performed by numerical simulation with Autodesk CFD software v.15.1. Results show that the temperature of the surface reaches values higher than the ones measured inside the sample holder. This difference of temperature is in good agreement with the visual observation of the phase transformations in the treated material, showing a simple and valuable tool to better control of plasma treatments.
\end{abstract}

Keywords: Ultimate surface temperature, $R F$ plasma treatment, Temperature simulation.

\section{Introduction}

It is already common knowledge, by thermodynamic laws and Arrhenius equation, that the material's temperature influences its reactivity with the environment, either in kinetic form (reaction rate), in the form of activation energy (heat required to allow the beginning of a reaction) ${ }^{1}$, surface temperature for processes like etch rate ${ }^{2}$, deposition rate ${ }^{3-5}$, or polymerization ${ }^{4,6}$ of the materials exposed to plasmas. However, there are cases in which is necessary to avoid the occurrence of specific reactions by controlling the reached temperature on materials surface, such as in food sterilization processing that degrades the flavor in higher temperatures ${ }^{7}$, controlled solidification ${ }^{8}$, precipitation of unwanted second phases in metal alloys, grain growing, and phase change in ceramics ${ }^{9}$. Therefore, many works were conducted with techniques that would allow the measurement of this variable in surface treatments, especially in plasma systems.

In cleaning and degradation plasma processes, where specific reactions of organic compounds depends on the temperature ${ }^{10,11}$, the correct analysis of this variable is very important. Previous works found that when stearic acid heats due to reactions with plasma, it melts and this leads to many undesirable effects, such as the formation of resistant compounds and boiling. It has already been evidenced that in relatively high temperatures $(313 \mathrm{~K})^{12,13}$, even when the material begins in the solid state, functionalization reactions becomes more important than degradation, leading to polymerization processes. On the other hand, treatments at lower temperatures $(273 \mathrm{~K})$ lead to high mass loss, where

* e-mail:mafra@utfpr.edu.br the possible by-products of functionalization are removed in degradation processes ${ }^{10}$.

On most studies of plasma degradation, the temperature measurement was obtained by a thermocouple, which is placed into the sample holder or into the sample itself. However, the exposed surface reacts with the plasma active species and could reach higher temperatures than those measured. Also, simply placing the tip of the thermocouple exactly on the surface is not an efficient method because plasma species could interact with the metallic tip, heating it more than the sample $\mathrm{e}^{14}$.

Knoerzer $^{7}$ examined many different temperature measurement techniques like thermocouple, resistance thermometer, thermal-paper (also found in ${ }^{15}$ and ${ }^{16}$ ), autoclave tape and a fiber-optic sensor. Among all these techiniques, he found that fiber-optic system was a reliable method since it did not suffer from the heating effect of charged particles from the discharge. However, some plasma reactors do not allow the easy placement of this kind of fiber inside the chamber.

Galvão $^{17}$ and Leal ${ }^{18}$ have extrapolated the surface temperature of a steel AISI M35, analyzing its microstructure and hardness along the thickness of this metal exposed to plasma. They concluded that on a microlayer of surface material, the temperature could be more than $100 \mathrm{~K}$ higher than the bulk.

Salter and Amiel ${ }^{19,20}$ have used infrared cameras to analyze the surface temperature of materials treated under special plasma and laser conditions. Salter ${ }^{19}$ observed that the desorption of organic molecules over a Si subtract are temperature-dependent, which is related to its own vapor pressure. Amiel ${ }^{20}$ observed that the measured surface 
temperature by IR camera was slightly higher than the one measured above the subtract with a thermocouple. However, not every system allows the visualization of a sample surface temperature and still needs other approaches to reach a correct value.

$\mathrm{Akamatsu}^{8}$ and $\mathrm{Osch}^{21}$ used experimental methods and numerical analysis to extrapolate values to extreme conditions of samples exposed to ion implantation and lasers, working with very high temperatures and shorter times. In those cases, errors of tens of degrees are easily acceptable. However, when working with cold plasma systems, this kind of error could mean very different material behaviors.

On most systems, the use of thermocouples to measure temperature inside a subtract is acceptable due to a good heat conductivity of the subtract and the simplicity of setup. On the other hand, when the heat conductivity of treated materials is low, as in polymer and other organic molecules processing, simply using a thermocouple inside a substact holder is not enough because these materials are very sensitive to small temperature variations on its surface.

Previous works on RF discharge by Farias $^{10}$, DC and after-glow by Bernardelli ${ }^{12,22}$ and Mafra ${ }^{13}$, used thermocouples inside the crucible and inside the sample, respectively. However, since reactions between sample and plasma generated species happen on the surface and the sample has a thickness of about a few millimeters, at least at the beginning of the treatment, there should be a delay on temperature measurements. Therefore, the present work present a way to achieve a most accurate value of surface temperature, by combining the thermocouple measurements into the sample and numeric simulation of the temperature at plasma-sample interface.

\section{Materials and Method}

\subsection{Experimental setup}

The experimental setup is depicted in Figure 1. Figure 1a shows the plasma reactor, which consists of a borosilicate tube with $34 \mathrm{~mm}$ inner diameter, $650 \mathrm{~mm}$ length and $2 \mathrm{~mm}$ thickness. The plasma is generated by a copper coil with 9 turns, connected to a radio frequency power source Tokyo Hi-Power, RF-300 model (and a matching box model MB$300)$, with a frequency of $13.56 \mathrm{MHz}$, which was used with the fixed power of $50 \mathrm{~W}$ (400 V peak-to-peak). The atmosphere is composed by a gas mixture of Ar- $10 \mathrm{vol} \% \mathrm{O}_{2}$ flowing at $750 \mathrm{sccm}$ at pressure of 1.5 Torr.

A temperature control system ${ }^{[1]}$ (TCS) consisting of water recirculation (pumped at $95 \mathrm{~L} / \mathrm{h}$ ), covering $110 \mathrm{~mm}$ of the discharge tube length, was used. The sample holder is made of poly-tetrafluoroethylene, and its design and dimensions are shown in Figure 1b. A $4 \mathrm{~mm}$ deep fitting groove is provided to place the sample. The curved face of the sample holder is shaped to allow good coupling between the sample holder and the glass tube. The work material was $95 \%$ pure Stearic Acid (S.A.), because it is a standard molecule to plasma degradation studies. It was placed on the sample holder in a molten state, until a small amount was above the surface. When solidified, the excess of S.A. was scraped with the aid of a metallic blade, to produce a flat surface. Due experimnental setup, it is not possible to move or visualize the sample during treatment as in many other plasma reactors. However, this material has a melting point at $341 \mathrm{~K}$ that will be used as a temperature indicator.

The thermocouple (Type K) can be placed at two different positions, always at $7 \mathrm{~mm}$ above the base of the sample holder (in central line), and penetrating the sample holder approximately $12 \mathrm{~mm}$, from the front or back sides. Due to RF field of plasma source, there was some interference in the thermocouple measurements. These were observed as a constant increase in reading value while the RF source remained on. After several adjustments in the configuration, position and electrical insulation of the equipment, the increment was reduced to differences smaller than $2 \mathrm{~K}$. After each experiment, when the RF source was turned off, the value of the difference in the temperature reading was noted and used to correct the data.

The sample holder was placed in the central position of TCS in all experiments. The model used for the simulation corresponds to a section of $10 \mathrm{~mm}$ length of the front and back positions of the sample holder, with the glass, as shown in Figure 1c.

Each experiment was repeated four times, and the error bars are calculated as a confidence interval of $95 \%$.

\subsection{Experimental and simulation setup}

The parameters of the experiments can be seen in Table 1 . In order to determine the contact resistance between the sample holder and the discharge tube (Condition 1), a sample was placed initially at $293 \mathrm{~K}$ in the central position of TCS, and the pressure was reduced to 0.01 Torr to inhibit thermal exchanges other than conduction. Heat exchange by radiation was not considered in this system due to the low temperature differences between sample and the TCS, and the short time for cooling or heating. In these experiments, the TCS contained water at temperatures of 273 and $313 \mathrm{~K}$ (for heating and cooling, respectively) and the evolution of the sample temperature was recorded every $1 / 3$ minute until stabilization. Simulations have shown TCR values of 0,007 and $0,013 \mathrm{~K} . \mathrm{m} / \mathrm{W}$, for heating and cooling, respectively.

To begin the experimental analysis (Condition 2), the temperature of the sample was controlled at 273, 293 or 313 $\mathrm{K}$ (stabilized for 30 minutes under vacuum), considering these values as the initial temperature. After that, the plasma was turned on and the temperature was measured at every 20 second up to 12 minutes of exposure. Because of different behaviors in the front and the back of the sample, analysis was made on both positions and the results were discussed. 


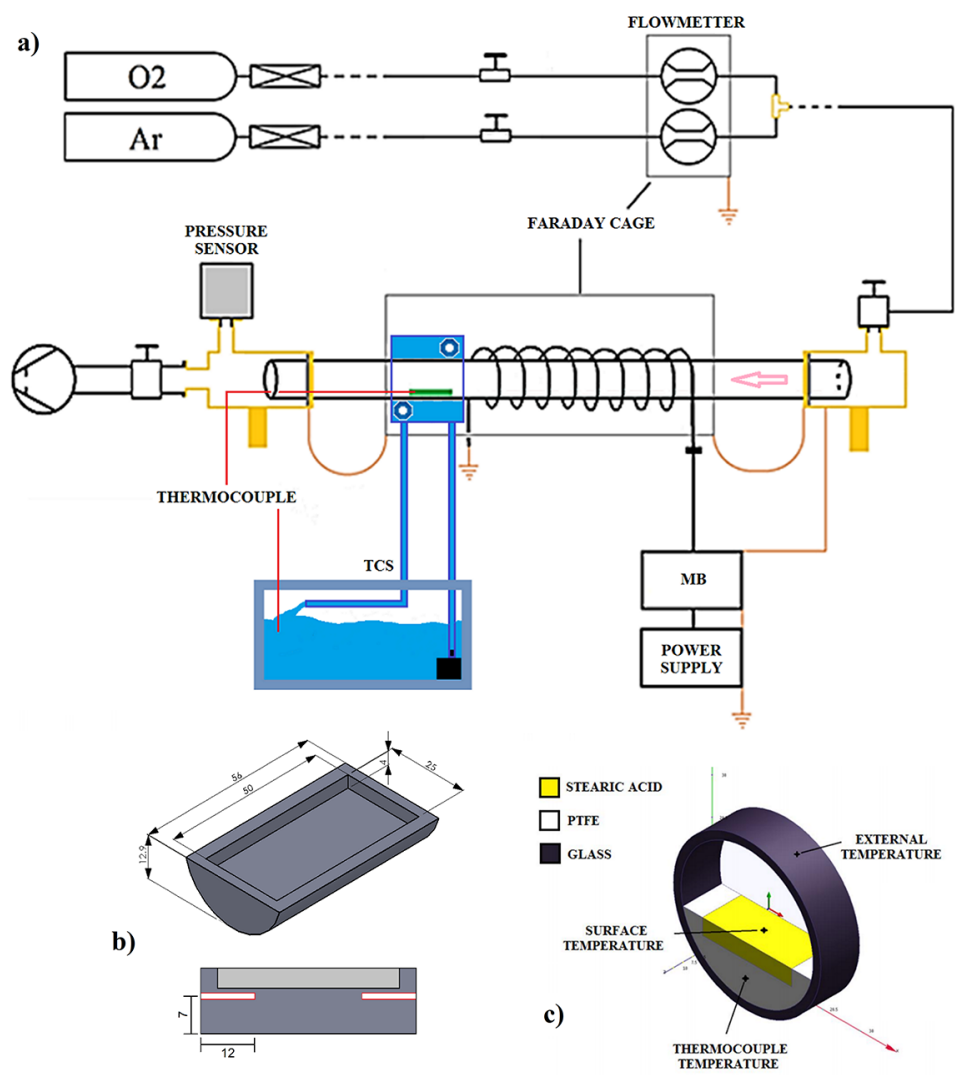

Figure 1. (a) Experimental Setup, (b) Detail of sample holder (dimensions in mm), (c) Three-dimensional model used for simulation

Table 1. boundary conditions for simulations.

\begin{tabular}{lccc}
\hline & Condition 1 & Condition 2 & Condition 3 \\
\hline Regime & Transient & Transient & Transient \\
Type & Experimental and Simulation & Experimental and Simulation & Simulation \\
Time (min) & $0-20$ & $0-12$ & $0-12$ \\
Position & Thermocouple & Thermocouple & Surface \\
TCS temperature.(fixed) (K) & $273 ; 313$ & & $273 ; 293 ; 313$ \\
Initial temp. (K) & 293 & $273 ; 293 ; 313$ \\
Heat generation & No & Yes \\
TCR (K.m.W W $^{-1}$ ) & Unknow & R1; RM; R2 \\
Number of samples & 4 & 4 in the front position 4 in the back position
\end{tabular}

R1, R2: thermal contact resistance values obtained by simulation 1 on back and front position; RM: average value of the thermal contact resistance R1 and R2

After the measurements, simulations were carried out, beginning from a system without heat generation on the surface, and by trial, adding heat generation values on the surface to fit the simulated curves to the experimental results.

Using the boundary values, heat generation on the surface, TCR and different initial temperatures (273, 293 and 313 $\mathrm{K}$ ) obtained under Condition 2, the temperature curve on the surface was extracted.

The values obtained are validated by two methods. First, since the melting temperature of S.A. is $341 \mathrm{~K}$, when the surface reaches this value, melting spots should appear on the surface. To observe these physical changes in the samples, a series of experiments were made, removing the samples from the reactor after 2, 4, 6, 8, 10 and 12 minutes of treatment, for each initial temperature.

The second approach to validate the simulation was the analyses of the temperature at two positions of the sample. Thus, a second thermocouple was placed inside the SA sample and the temperature profile was measured and simulated. 


\subsection{Material data}

Table 2 lists the properties of materials used for the simulation.

Table 2. Data of materials used ${ }^{23,24}$.

\begin{tabular}{lccc}
\hline & $\begin{array}{c}\text { Thermal } \\
\text { Density } \\
\left(\mathrm{g} / \mathrm{cm}^{3}\right)\end{array}$ & $\begin{array}{c}\text { Conductivity } \\
\text { Coefficient } \\
\left(\mathrm{W} \cdot \mathrm{m}^{-1} \cdot \mathrm{K}^{-1}\right)\end{array}$ & $\begin{array}{c}\text { Melting } \\
\text { Temperature } \\
(\mathrm{K})\end{array}$ \\
\hline $\begin{array}{l}\text { Glass } \\
\text { (borosilicate) }\end{array}$ & 2,7 & 1,10 & - \\
$\begin{array}{l}\text { PTFE } \\
\text { S.A. }\end{array}$ & 2,16 & 0,25 & 600 \\
\hline
\end{tabular}

\subsection{Boundary conditions and considerations}

All simulations were performed using a simple transient thermal conduction model with contact resistance between sample holder and glass, at Autodesk CFD simulation software, version 15.1. This software uses a model called Compact Thermal Model that uses finite elements nodes to calculate transient heat transfer. In software, only material properties and boundary conditions were input while all specific variables of simulation were kept as default of software.

The meshing were created with at least 7500 nodes distributed over the model. The simulation was created with at least 3 interactions per second, and data was saved at each 5 second. All simulated data was extracted direct from the software, by selecting a point of interest and extracting the evolution of the temperature on that point.

All simulation were purely heat transfer by conductivity, without considering radiation (due low temperature diference between sample and environment) and convection (vacuum environment) heat transfer modes. In the used software, it was not possible to consider phase changing of material and so, absolut values of surface temperature after melting of material may not be correct, but should be close enough. This phase changing of material will be used as a temperature indicator.

As is the objective of this paper, the surface temperature will be an extrapolated value from simulation, based on what was designed to obtain a heating profile at thermocouple position.

\section{Results and Discussion}

\subsection{Experimental measurements during plasma treatment}

Experimental results are shown in Figure 2, to the front (a) and back (c) positions, also it shows the temperature variation rate (measured and simulated) for front (b) and back (d) positions. Comparing Figures 2 (a), and (c), it is possible to observe that the front position was more heated than the back position to all respective onset temperatures. The highest heating on the front position was $26 \pm 3 \mathrm{~K}$, while on the back position, it was $13 \pm 2 \mathrm{~K}$. This difference could be related to a higher consumption of reactive species at the front position, leaving a lower concentration to interact with the surface at the back position, according to the gas flow.

The temperature at the front position of the sample with onset at $313 \mathrm{~K}$ showed values almost equivalent between minutes 4 to 6 of treatment. It can be attributed to the sample melting in a position close to the thermocouple. If the SA properties are considered, Falleiro ${ }^{25}$ has shown that melting temperature of this substance is almost constant in relation to the pressure inside the vacuum chamber. On the other hand, Belmonte ${ }^{26}$ have observed the melting of hexatriacontane and stearic acid samples exposed to $\mathrm{Ar}-\mathrm{O}_{2}$ afterglow and, under his conditions, melting was attributed to physical-chemical transformation reactions leading to changes in the nature of the sample. Indeed, these transformations also occurs in the present work, as will be presented later.

In almost all cases, it takes approximately 2 minutes to increase the temperature of the thermocouple in $2 \mathrm{~K}$. However, as it will be seen later on this paper, this amount of time is enough to produce molten material with onset of $313 \mathrm{~K}$, as indicative of a considerable higher surface temperature and/or structural and chemical transformations of the sample with the plasma exposition.

To highlight small changes in the heating process that are not clearly visible in a temperature vs. time curve, derivative of temperature with time was used to reproduce the experimental measurements, visible at Figures 2 (b) and (d). In a thermal system based on conduction with heat generation, the existence of different profiles of heating rate ( $\mathrm{dT} / \mathrm{dt})$, during the treatment, indicate the occurrence of different phenomena or change in heat generation. To the sample at onset of 273 $\mathrm{K}$, in both front and back positions, the heating rate rapidly increase until it reaches a maximum value at approximately 3 minutes of treatment, decreasing slowly after that.

In an ideal system, if no physical-chemical modifications occur, a constant temperature profile could be reached after a long time of treatment, and the heating would reach $\mathrm{dT} / \mathrm{dt}=0$. If on the back position this tendence is observed, in front position, from 6 to 10 minutes of treatment, the heating is almost constant. It means that something had changed on the surface and more heat is being produced at this point. This behavior could be related to the own heating of the sample, which favors the reactivity and, therefore, it increases the heat generation and transfer.

At $293 \mathrm{~K}$, there is also a rapid heating at the beginning, but it lasts until 4 minutes on the front position and until 2 minutes on the back. At the front position, there is a peak of heating that last only a few seconds. Since this event happened to all samples under this condition at the same period, this outlier point was considered on the simulation. Two possible explanations were found to account for this behaviour. First, Bernardelli ${ }^{12}$ showed that atomic oxygen 
(a) Front

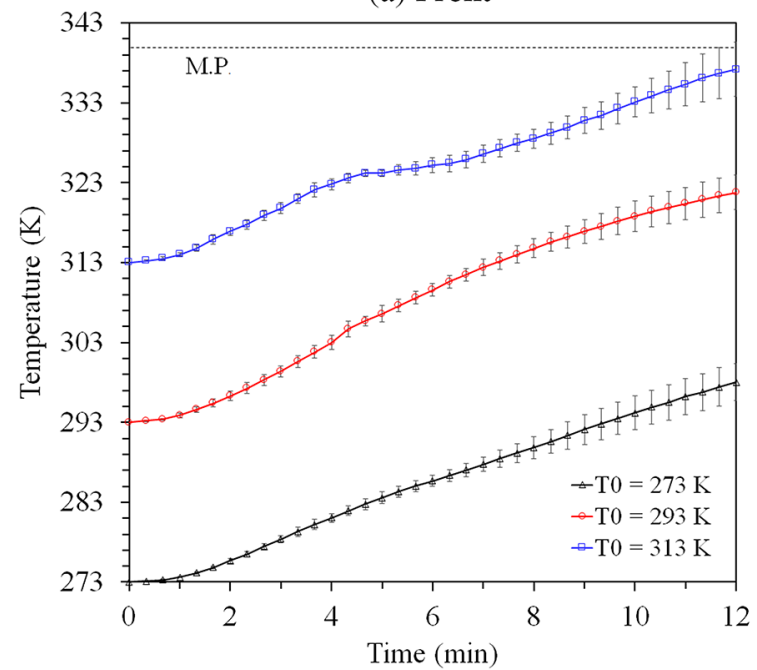

(c) Back

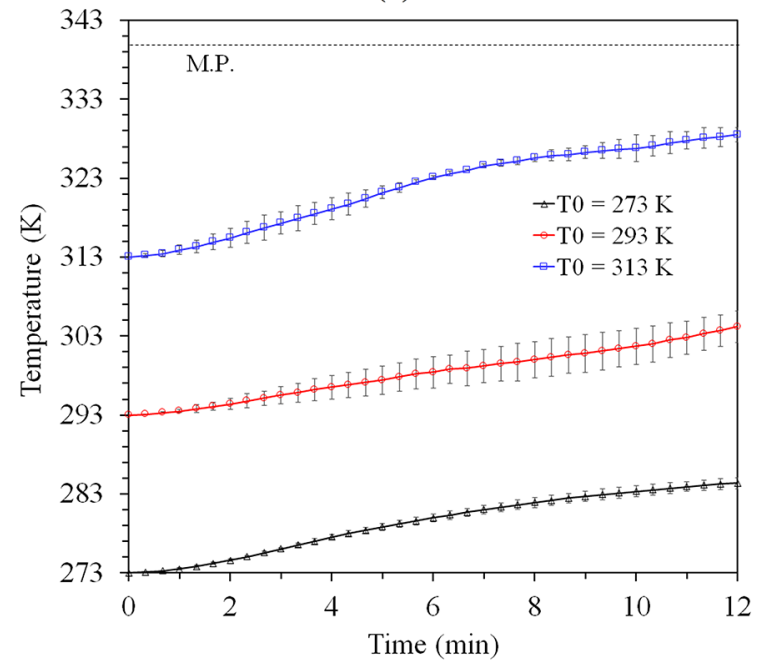

(b) Front

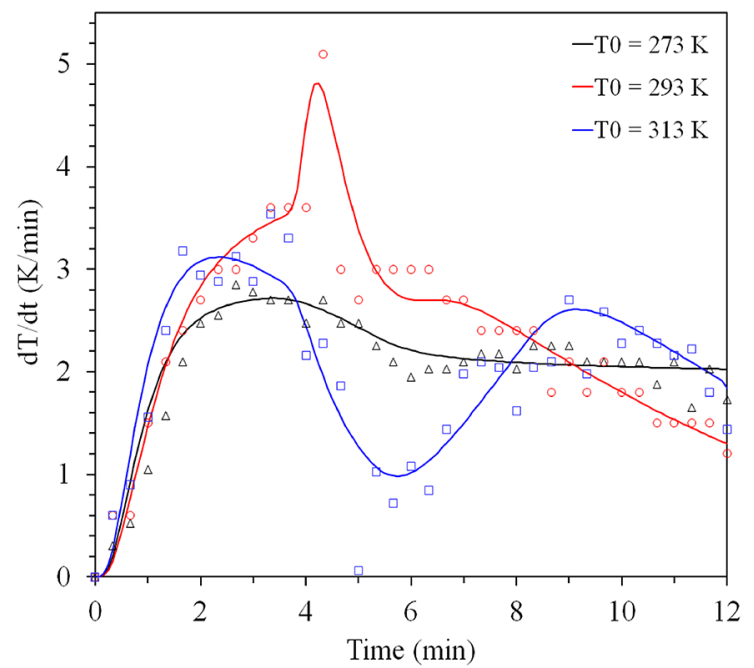

(d) Back

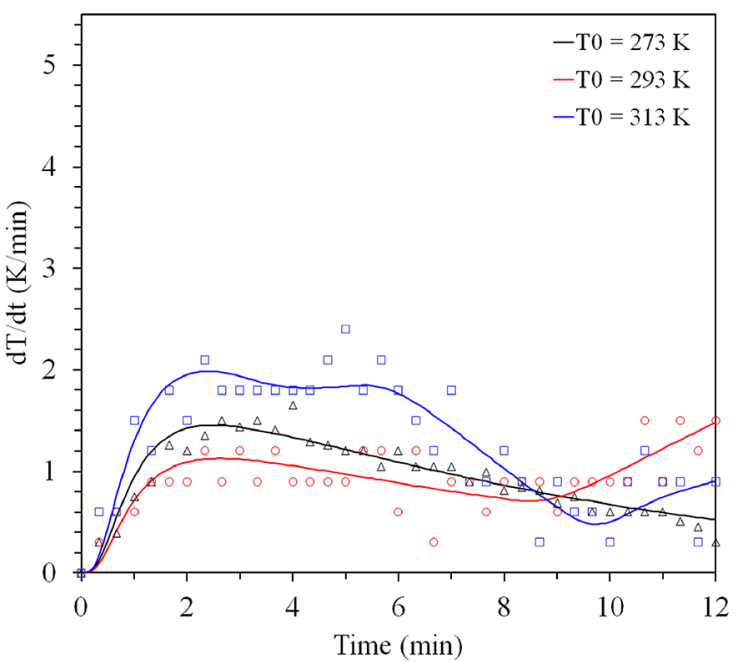

Figure 2. Experimental measurements of temperature in the region of thermocouple for the front position (a) and its instant variation measured (signals) and simulated (line) (b); measured temperature in the backt position (c) and its instant variation measured (signals) and simulated (line) (d). The dashed line indicates the value for the melting temperature (M.T.) of S.A.

active species are able to diffuse during liquid phase, and promote reactions, increasing the heating of the sample at the moment. At the same time, Tagawa ${ }^{27,28}$ found that when polymers are exposed to atomic oxygen and UV light, the angle of incidence can increase the rate of interaction considerably. However, since the increase on the incident angle due the melt puddle of SA is minimal on experimental conditions, the effect of the presence of a liquid phase on SA is considerably more important to the heating.

After this peak at $293 \mathrm{~K}$, the heating slow down to a plateau where then it is kept constant for approximately 1 minute, and then it starts to slowly decrease until the end of treatment. After a long exposure with the material on liquid phase (about 40 minutes), Mafra ${ }^{13}$ and Bernardelli ${ }^{12}$ observed a decrease on the sample temperature due to oxygen saturation of the molten puddle and structural changes on the treated material. It is worth to remember that in the present work the sample was immersed in the discharge, thus it is submitted to the impingement of charged species. These collisions can heat the sample and can produce chain scission, which also contributes to the occurrence of liquid phase.

In the back-side profile with onset 293K, between 2 and 10 minutes, there are lower variations on heating when compared with onset at $273 \mathrm{~K}$, meaning that heating on sample surface should be increasing with time and temperature. Otherwise, the heating should decrease with time, as an ideal system should look like. However, after 10 minutes is observed an increase on the heating, resulting from the presence of liquid 
phase on the surface, which will be better understood from the visual analysis presented later.

At $313 \mathrm{~K}$ there are no visible peaks of heating that could be related to the melting of the sample. However, individually analyzing experimental data from all samples at $313 \mathrm{~K}$, there was a small peak of heating for each sample, but smaller, and at different times, which were masked by data averaging and it is the result of a more aggressive treating condition. As it will be seen later, it is again related to the beginning of the sample's melting. Between 3 and 5 minutes, on the front position, heating decreases rapidly, almost reaching $0 \mathrm{~K} / \mathrm{min}$ at 5 minutes; this behavior was associated to the melting of the material around the thermocouple. After this point, the front position heats again, reaching a second maximum value at 9 minutes and only decreasing after that. On the back position, after the maximum value at 3 minutes, the system keep a constant heating until 6 minutes and it starts to decrease until the end of experiment.

An interesting observation about the simulation process relates to the generated heat to all samples. A main result obtained by the successive approximations to achieve the best fit is that any modification on the heating measured on the thermocouple, in this system, happened on the surface, between 40 seconds to 1 minute before the event. As an example, the heating peak at the front position with onset at $293 \mathrm{~K}$, happened at 4 minutes and 20 seconds while on simulation, the generated heating peak had to happen at 3 minutes and 40 seconds on the surface to reproduce a peak at the thermocouple position. Thus, it is reasonable to say that, on this kind of system, there is a delay between the surface event and the response on the measuring system as it should happen on others systems that uses thermocouples under sample holder.

\subsection{Simulation on the surface}

Figure 3 shows the simulated temperature profiles of a selected point on the surface, located on the central axis of the sample to the front ( $3 a$ ) and back ( $3 b)$ posiotions. In all cases, simulation shows that the temperature increases very quickly on surface when compared with the measured point (on the thermocouple). This high heating rate is a situation that is already known when treating organic molecules in oxygenated plasmas, as shown by Praveen et. $\mathrm{al}^{29}$ on their work; even a short exposure to oxygen plasmas, of less than 1 minute, was enough to heat lignocellulosic natural coir fibers to a temperature where it degrades. On this study, the amount of power and oxygen concentration applied were considerably lower, and also, the TCS system provided an efficient way to heat the extraction of sample, lowering the material's reactivity with plasma and, then, making it possible to analyze the temperature curve.

In all cases, the measured temperature on thermocouple takes more than 2 minutes to increase approximately $2 \mathrm{~K}$, while the simulation shows that, on the surface, it takes 6 seconds (or less) to increase the same amount. This is obviously related to the fact that the only source of heating of this system is located at the surface (by the interaction plasma-sample), and the low thermal conductivity of both the crucible and the sample.

Absolute values of temperature variation at the end of simulation are 73,77 and $74 \mathrm{~K}$ at the front, and 29,38 and $41 \mathrm{~K}$ at the back for the onsets of 273, 293 and $313 \mathrm{~K}$, respectively. Those values are much higher than the measured ones on the thermocouple, meaning that in treatments of organic materials in oxidative plasmas, a great attention need to be devoted to temperature measurements, to avoid undesirable reactions and/or misinterpretations of results. This high temperature is in accordance with the results found by $\mathrm{Mafra}^{30}$, where exothermal reactions between $\mathrm{Ar}-\mathrm{O}_{2}$ late afterglow were strong enough to heat the gas downstream. In the present work, where the sample is placed into the discharge, and, because of the energetic collisions that will take place, a more important heating is expected.

At the front position, all samples achieved on the surface, temperatures that are higher than the melting of SA. This occurred at $10 \mathrm{~min}$ and $40 \mathrm{sec}$ to sample with onset at $273 \mathrm{~K}, 4$ minutes for onset of $293 \mathrm{~K}$ and at 50 seconds to the onsets of $313 \mathrm{~K}$. In respect to the derivate of those curves, it is visible that the higher it is the onset, the faster it increases its temperature with time, at least on the first minute of exposition.

It is also observable that the heating peak at the front position of the sample with onset at $293 \mathrm{~K}$ takes place right after its surface reaches the SA's melting temperature. One could relate this phenomenon with the melting of the sample surface, due to previous explanations that the reactivity of the material increases with the formation of the liquid phase. However, this condition only appears with the onset of 293 $\mathrm{K}$, and under other conditions, it is believed that there was not a good balance of heat conduction and heat generation to create a visible heating peak. At $273 \mathrm{~K}$, the melting only occurs after 10 minutes and, at that time, the heat generated is already well distributed through the sample and a heating peak was not well detected. At $313 \mathrm{~K}$, the reactions were really fast and this creates a condition where the heat peak happened but changed considerably to each sample, and it was masked due to averaging between the other samples.

With the onset of $313 \mathrm{~K}$ and at the front position, there is a moment, at 3 minutes and 30 seconds, where the temperature of the surface decreases for a brief moment. It was stated that the decrease on the generated heat under this condition was related to the melting of the sample on a region close to the measuring point. In order to verify this, a point close to the interface between SA and PTFE was selected, right above the thermocouple position, and the temperature vs. time curve was simulated, as shown in Figure 4. 
(a) Front.

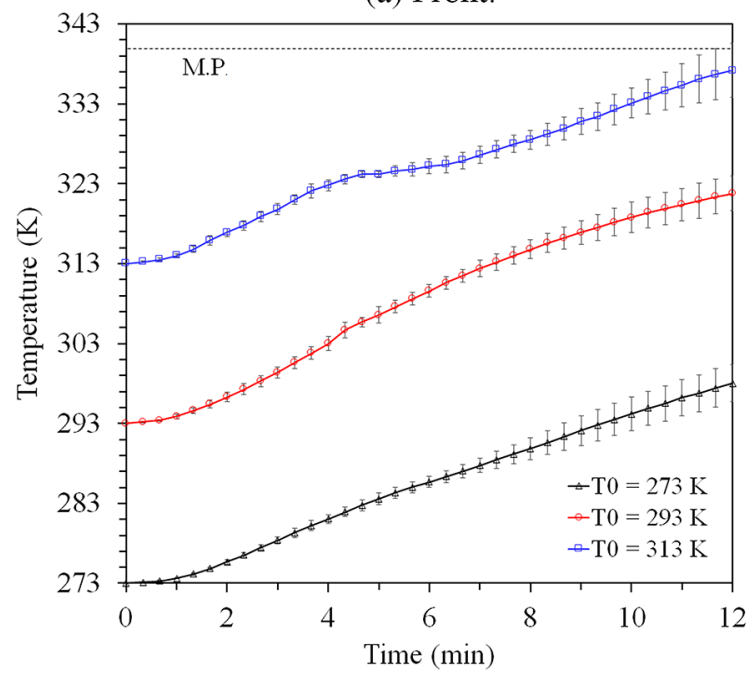

(b) Back

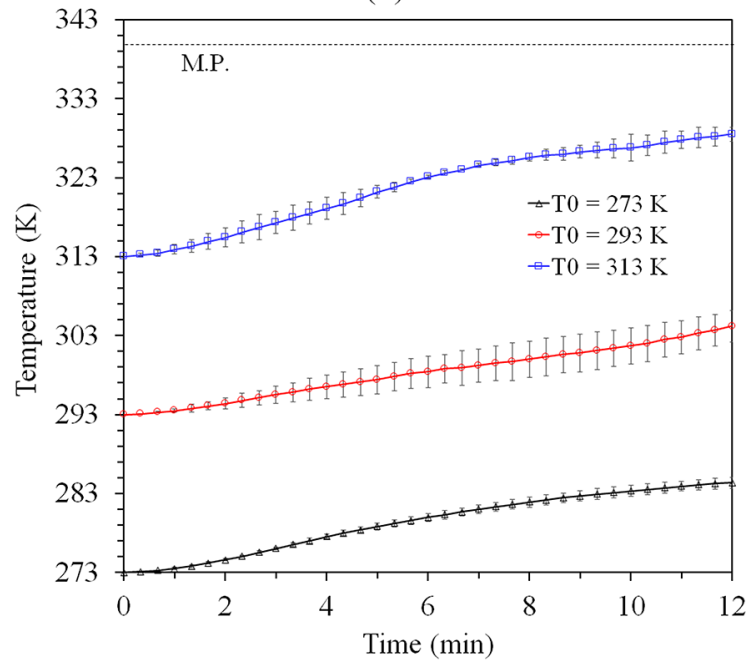

Figure 3. Temperature simulation on the surface at the front (a) and back (b) positions, in relationship to the gas flow. The dot line represents the melting temperature of the sample.

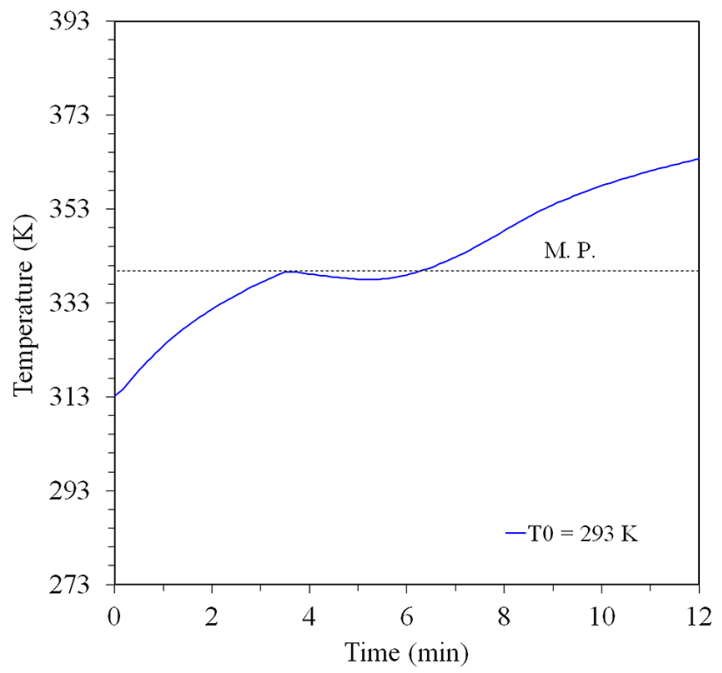

Figure 4. Simulated temperature for the interface region between SA and the sample holder for the front position with onset at 313 $\mathrm{K}$. The dot line represents the melting temperature of the sample.

In accordance with the hypothesis, the temperature on this region was kept close to the melting temperature of SA during the entire time that the generated heat in this condition remained at its lowest plateau. Therefore, the latent heat of fusion is a very important variable to consider at simulation. However, due to software limitations, it was not possible to consider this variable. For this reason, the absolute temperature of the surface after melting must be measured with caution.

On the back position, the only condition that exceeds the M.T. line is the one with onset at $313 \mathrm{~K}$ at 2 minutes and 20 seconds. The increase on the heating of the onset at 293 $\mathrm{K}$ after 8 minutes, at a temperature considerably lower than the melting point, is not related to the phase transformation of the surface, with another source of heat being necessary to produce this phenomenon.

\subsection{Validation of simulation 1 (visual analysis)}

Samples from this topic were prepared only after the results of simulations had been completed. Thus, the results of this topic serve to validate the simulation related to with when and where melting of surface would occur. Figure 5 shows the results.

Starting with sample with onset at $273 \mathrm{~K}$, after 10 minutes of treatment, a small puddle of molten material appear on the surface of the front position. Later on, this puddle only increases in size. This result is rigorously in accordance with Figure 3 (a) for the same onset. On the back position, no sign of melting appears on the surface, also in agreement with Figure 3 (b) for the same onset. It was also observable, that after 6 minutes of treatment, there is a weak yellowing of the surface (not so visible on the pictures due to light effects of the camera), that is a clear sign of chemical modifications of the organic compound due oxidation from plasma $a^{31,32}$.

Next, to samples with onset at $293 \mathrm{~K}$, the first picture at 2 minutes do not show any sign of melting on the surface in accordance with Figures 3 (a) and (b). However, at 4 minutes there is an already large puddle of melting material at the front position, while the back position is still solid, also in accordance with Figures 3 (a) and (b). A sample was made with this same onset, but was removed from reactor at 3 minutes and there was a small puddle of molten material, similar to the one with onset at $273 \mathrm{~K}$ after 10 minutes of exposure. On simulation, melting would only occur at 3 minutes and 50 seconds, but it still close enough to be considered a valid result. Again, it is worth to say that physical-chemical 


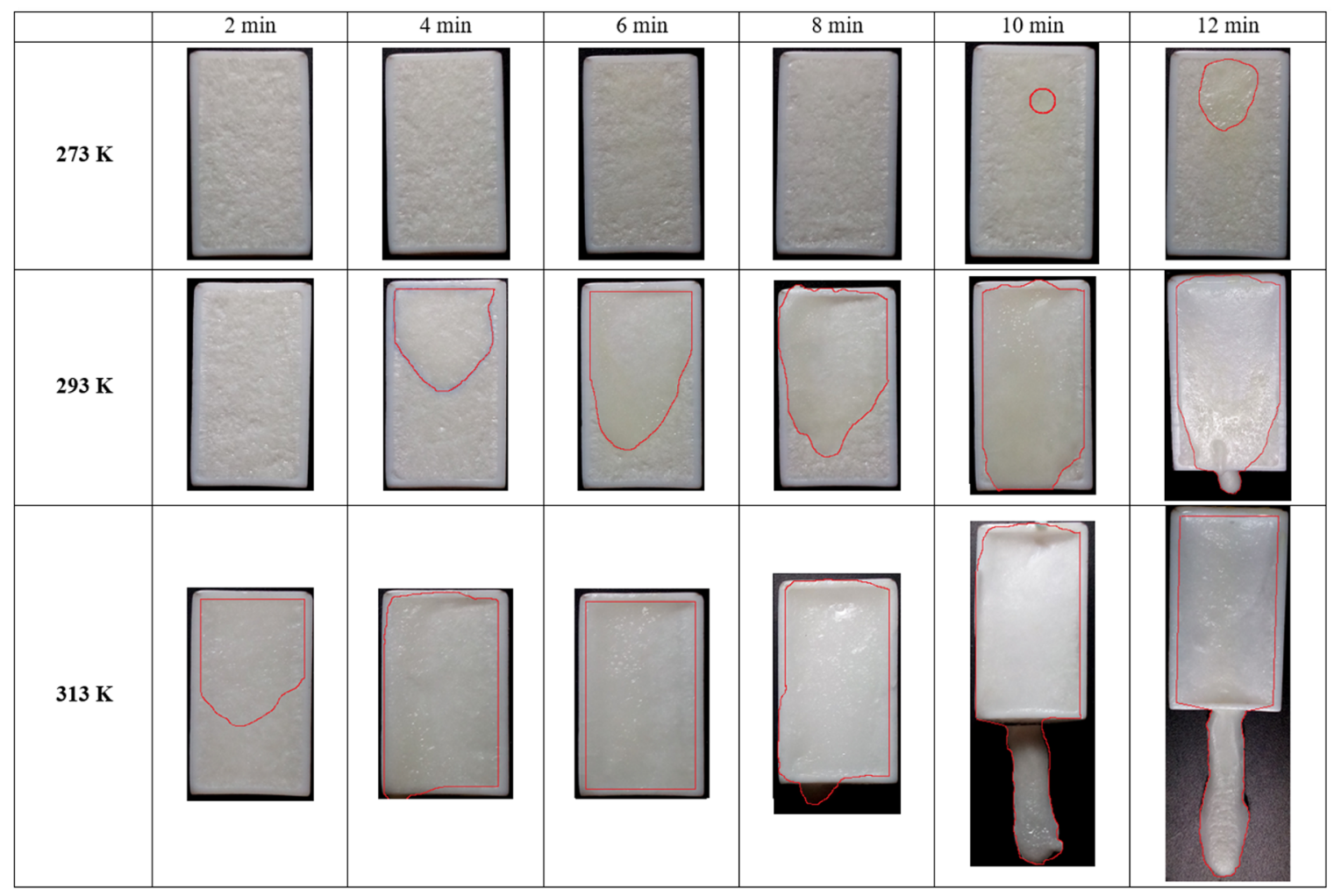

Figure 5. Sample images during experiment at different onset temperatures. Sample positioning on picture is up $=$ front position, down $=$ back position, relative to gas flow.

phenomena, which will certainly happen, can also contribute to the appearance of the molten material.

Looking at the pictures in Figure 5, with onset at 293 $\mathrm{K}$, one could believe that after 6 minutes there was already molten material on the surface of the back position. However, after a close inspection on the surface, one could observe that the molten material is actually from the front position. This liquid material was dragged by the flow, covering the back position.

Using a schematic drawing, in Figure 6, a clear explanation follows. Six different moments (named A to F) are identified. At moment A, the sample was still completely solid. At moment $\mathrm{B}$, a small puddle of molten material appears on the surface of the front position as similar as what happens with onset at $273 \mathrm{~K}$ and 10 minutes, and at $293 \mathrm{~K}$ and 3 minutes. While the puddle grows in size and depth, on moment $\mathrm{C}$, it starts to be pushed towards the back position, due to the gas flow, as can be seen at moment $\mathrm{D}$, and at $293 \mathrm{~K}$ and 6-8 minutes.

Then, after this molten material reaches the back position, it covers it, just like what is seen at $293 \mathrm{~K}$ and 10 minutes. However, the simulation on the back position predicts that the surface at that time did not reached the melting temperature of the sample yet.

Looking at Figure 6, the simulation results of the back position with onset at $293 \mathrm{~K}$ can be more clearly understood.
As shown at moment $\mathrm{E}$, the molten material cover the back position, but without changing the previous state of the surface material considerably. This observation is particularly interesting, because the aforementioned physical-chemical reactions indeed are playing a role, and consequently, the molten material is no longer the initial SA, but the by-product of its degradation on the plasma, resulting in a liquid puddle at a temperature lower than the SA's melting point. When it occurs, this puddle becomes a source of heat to the surface, increasing the temperature measured.

After some time, the amount of molten material that flowed to the back becomes so large that it leaks out from the crucible, as represented in Figure 6 at moment $\mathrm{F}$, as visible in Figure 5 with onset $293 \mathrm{~K}$ after 12 minutes, and onset at $313 \mathrm{~K}$ after 8 minutes.

Unfortunately, this pushing and leaking of material changes the heat profile of the system and the software used is not capable of predicting this kind of behavior. Therefore, once again, the absolute temperature of the surface after its melting must be taken into consideration due to this condition.

Finally, sample with onset at $313 \mathrm{~K}$ already shows a large puddle of molten material on the front position at 2 minutes. Looking to the size of the puddle and the previous conditions, it is possible to believe that it starts as a small puddle in 1 minute or less, in accordance with Figure 3 (a), 


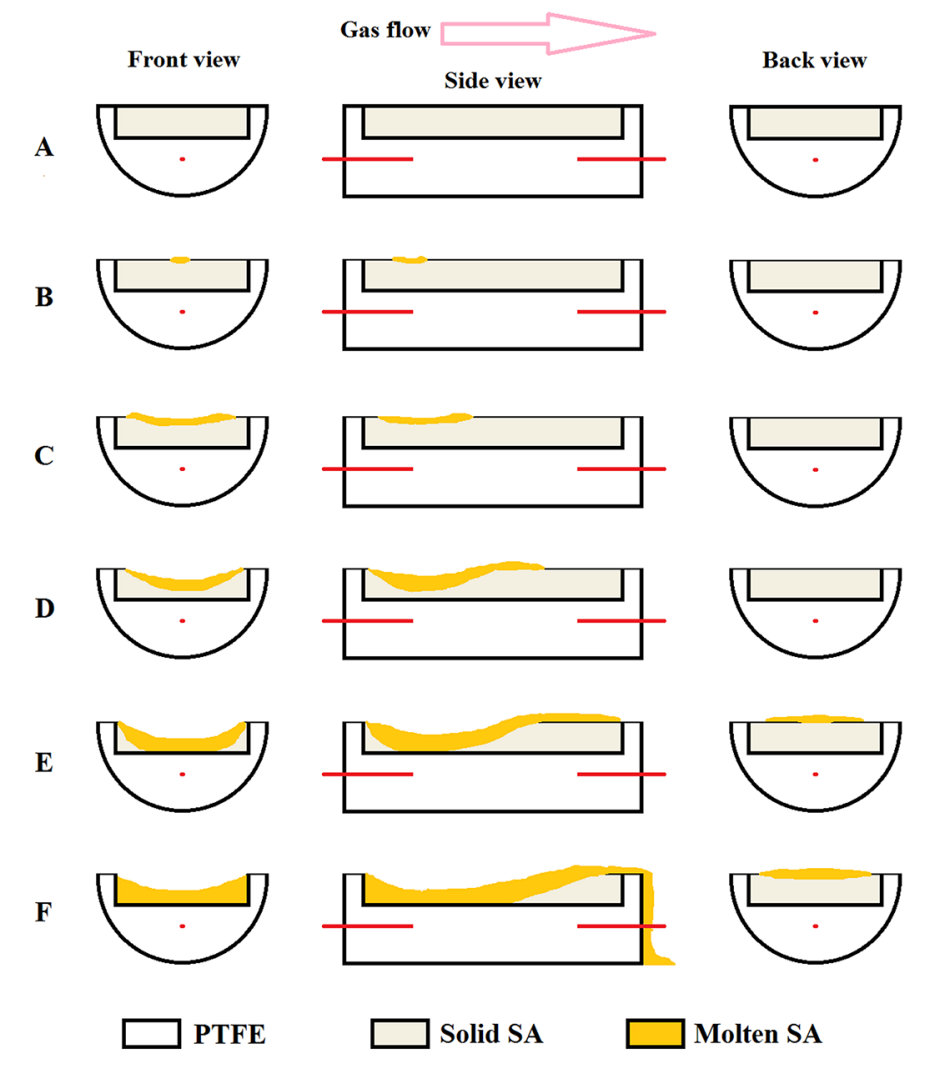

Figure 6. Schematic profile of sample during experiment. The red line represents the thermocouple and the letters represent different moments of experiment.

which shows that the melting would occur near 50 seconds of treatment. In addition, the back position do not show any sign of melting, in accordance with Figure 3 (b). The simulation on the back position predicts that melting would occur at 3 minutes and 20 seconds. Due to the rapid growth of the front molten puddle, it is possible that the surface of the back position reaches the melting temperature close to the predicted time, while it is been covered by molten material from the front position.

In this case, of onset at $313 \mathrm{~K}$, the back position shows that after 12 minutes, a great amount of material leaked out from the sample holder, greatly compromising the absolute value of temperature on the surface after melting values.

Another interesting point with onset at $313 \mathrm{~K}$ is that in the back position in Figure 6 (b), the temperature on the thermocouple was close from the M.T. line, while the surface should be molten, as predicted by Figure 3 (b). After the removal of the sample holder from the reactor, after 12 minutes of treatment, visual inspection reveals that most part of the sample is molten, except for a small part on the back of the back position, which remained solid, also represented in Figure 6 at moment $\mathrm{F}$ for the same position.
Meister ${ }^{33}$ worked with a very similar approach between simulation and experimental analysis, finding a good fit among his results. However, as in other works ${ }^{8,21}$, those temperature values obtained on the treatments were considerably high (thousands of $\mathrm{K}$ ) and variations of tens of degrees between simulation and experiment are usually acceptable. On most cases, these errors could be assigned to the measuring system itself.

On the other hand, in the present work, it was possible to achieve a higher accuracy on the results, allowing the prediction of when and where a phase-change transformation would occur by heating on sample surface (due interaction with plasma species), in a temperature range of less than 10 $\mathrm{K}$. On an industrial level, when plasma treatment is applied in temperature-sensitive materials like food ${ }^{7}$, other organic materials ${ }^{12,13}$, films deposition on surfaces ${ }^{34,35}$ or etching ${ }^{2}$, a good knowledge of the real surface temperature is very important for treatment control.

Therefore, the results obtained in this work showed how to approach, by simulation, a value of surface temperature on plasma treatments when the direct measurement of the sample's surface is not possible. 


\subsection{Validation of simulation 2 (second thermocouple)}

A second approach to validate the simulation procedure was to insert a second thermocouple inside the SA sample. However, this condition brings several complications to the experiment. Firs, positioning of the thermocouple on SA must be done while at liquid state during sample preparation and, thus, it makes it very difficult to reproduce in each sample the exact position of the measurement tip. Even little displacements on the position of the tip lead to very different temperature profiles during treatments. Secondly, pasting the tip on an exactly position was not a possible approach because normal adhesives do not adhere efficiently to a PTFE sample holder. Thirdly and lastly, when SA melts around the thermocouple tip during plasma treatment, atomic oxygen that was dissolved on liquid SA found a surface to recombine, which released heat and makes the measurements after melting even less trustable.

Knowing that, the decision was to do the simulations based on a single sample that best reproduced the previous experiments. The results are visible in Figure 7. The selection of onset at $273 \mathrm{~K}$ for this experiment is due a lower and more stable heating of the sample, which makes it easier to recreate on the simulation program. The positioning of the thermocouple tip was about $2 \mathrm{~mm}$ below the SA surface, which helps to avoid the melting of SA around the tip during experiment, while on the surface it melts.

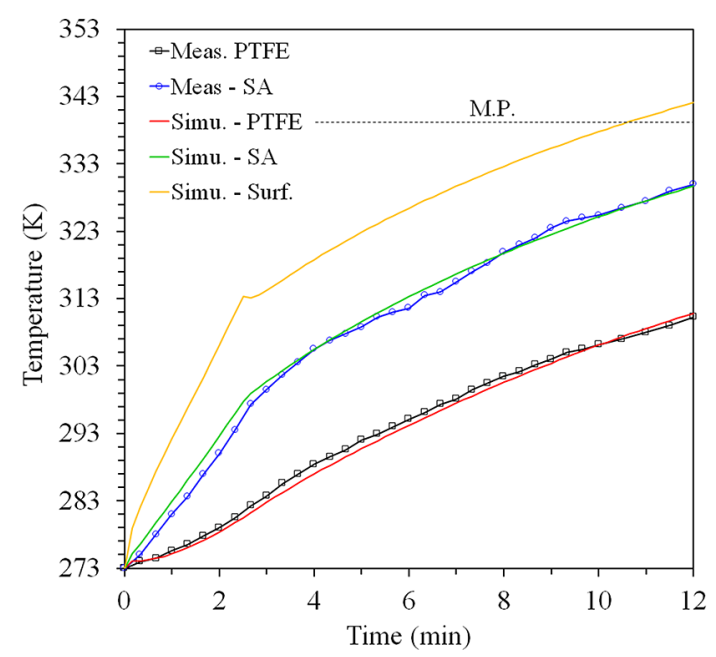

Figure 7. Experimental and simulated temperature with two thermocouples on front position with onset at $273 \mathrm{~K}$. The dot line represents the melting temperature of sample material.

Following the same route for experimental and simulation values, it was possible to verify that as expected, inside SA, the temperature rises faster than inside the sample holder, but follows a very similar pattern when compared to the one below it. At the surface, the melting temperature of the
SA is reached after 10 minutes of plasma exposure, similar of what was expected by the simulation. These results are in agreement with the visual aspect of the sample surface in Figure 5 for the same onset. However, even when the surface melts, some amount of SA remains solid around the thermocouple tip.

\section{Conclusion}

The simulation tool proposed here was able to successfully predict several conditions related to thermal phenomena occurring on the degradation process of an organic material by plasma. It was observed that organic compounds exposed to an oxidative plasma react in such a manner that produce several transformations in the material. Immersed in plasma, chemical and physical effects can plays important role on this heating, which is enough to melt the material in seconds of treatment, even when the temperature measurement was considerably different from the melting point. In addition, it was observed that when the sample reaches the liquid phase, it increases the heating by the enhancement of the interaction with the plasma. Even with the used software limited to solid state samples, it was possible to determine when the surface melting processes would occur on the material treated with good agreement to the observed transformation. Thus, the simulation presented here can be a valuable tool to basic understanding of reactions and transformations on the surface of organic materials in plasma treatments.

\section{Acknowledgement}

Authors kindly acknowledge the support given by Brazilian agencies of technological and scientific research: Fundação Araucária (FAPPR) proj. 25288-12/2011; CNPq projects 479593/2012-4 and 308798/2012-0; and CAPES by C. E. Farias grant.

\section{References}

1. Gijsman P, Hennekens J. The influence of temperature and catalyst residues on the degradation of unstabilized polypropylene. Polymer Degradation and Stability. 1993;39(3):271-277. DOI: 10.1016/0141-3910(93)90001-Y

2. Lee M, Lee WJ. The effect of substrate temperature on the etching properties and the etched surfaces of magnetic tunnel junction materials in a $\mathrm{CH} 3 \mathrm{OH}$ inductively coupled plasma system. Applied Surface Science. 2012;258(20):8100-8108. DOI: 10.1016/j.apsusc.2012.05.003.

3. Rai VR, Vandalon V, Agarwal S. Influence of surface temperature on the mechanism of atomic layer deposition of aluminum oxide using an oxygen plasma and ozone. Langmuir. 2012;28(1):350357. DOI: $10.1021 / \mathrm{la} 201136 \mathrm{k}$ 
4. Thiry D, Aparicio FJ, Laha P, Terryn H, Snyders R. Surface temperature: A key parameter to control the propanethiol plasma polymer chemistry. Journal of Vacuum Science \& Technology A: Vacuum, Surfaces, and Films. 2014;32(5):050602. DOI: 10.1116/1.4890672

5. Mutsukura N, Saitoh K. Temperature dependence of a-C:H film deposition in a $\mathrm{CH} 4$ radio frequency plasma. Journal of Vacuum Science \& Technology A: Vacuum, Surfaces, and Films. 1996;14(4):2666. DOI: 10.1116/1.579999

6. Nelson CT, Overzet LJ, Goeckner MJ. Role of surface temperature in fluorocarbon plasma-surface interactions. Journal of Vacuum Science \& Technology A: Vacuum, Surfaces, and Films. 2012;30(4):041305. DOI: 10.1116/1.4729445

7. Knoerzer K, Murphy AB, Fresewinkel M, Sanguansri P, Coventry J. Evaluation of methods for determining food surface temperature in the presence of low-pressure cool plasma. Innovative Food Science \& Emerging Technologies. 2012;15:23-30. DOI: 10.1016/j.ifset.2012.02.008

8. Akamatsu H, Yatsuzuka M. Simulation of surface temperature of metals irradiated by intense pulsed electron, ion and laser beams. Surface and Coatings Technology. 2003;169-170:219222. DOI: $10.1016 / \mathrm{S} 0257-8972(03) 00083-5$

9. Shackelford JF. Introduction to Materials Science for Engineers. 8th ed. London: Pearson; 2014.

10. Farias CE, Bianchi JC, de Oliveira PR, Borges PC, Bernardelli EA, Belmonte T, et al. Evaluation of sample temperature and applied power on degradation of stearic acid in inductively coupled radio frequency plasma. Materials Research. 2014;17(5):12511259. DOI: $10.1590 / 1516-1439.270714$

11. Belkind A. Plasma cleaning of metals: Lubricant oil removal. Metal Finishing. 1996;94(7): 19-22. DOI: 10.1016/00260576(96)81354-7

12. Bernardelli EA, Belmonte T, Duday D, Frache G, Poncin-Epaillard F, Noël C, et al. Interaction Mechanisms Between Ar-O2 PostDischarge and Stearic Acid I: Behaviour of Thin Films. Plasma Chemistry and Plasma Processing. 2010;31(1):189-203. DOI: 10.1007/s11090-010-9263-2

13. Mafra M, Belmonte T, Poncin-Epaillard F, Silva Sobrinho AS, Maliska A. Role of the Temperature on the Interaction Mechanisms Between Argon-Oxygen Post-Discharge and Hexatriacontane. Plasma Chemistry and Plasma Processing. 2008;28(4):495-509. DOI: 10.1007/s11090-008-9140-4

14. Piejak R, Godyak V, Alexandrovich B, Tishchenko N. Surface temperature and thermal balance of probes immersed in high density plasma. Plasma Sources Science and Technology. 1998;7(4):590-598. DOI: 10.1088/0963-0252/7/4/016

15. Sahin HT, Manolache S, Young RA, Denes F. Surface fluorination of paper in CF4-RF plasma environments. Cellulose. 2002;9(2):171-182.

16. Manory RR. A simple method for monitoring surface temperatures in plasma treatments. Journal of Vacuum Science \& Technology A: Vacuum, Surfaces, and Films. 1986;4(5):2392. DOI: 10.1116/1.574085

17. Galvão NKAM, Costa BLS, Mendes MWD, de Brito RA, Souza CF, Alves C. Structural modifications of M35 steel submitted to thermal gradients in plasma reactor. Journal of Materials Processing Technology. 2008;200(1-3):115-119. DOI: 10.1016/j. jmatprotec.2007.08.058
18. Leal EAD, Souza SIS, Alves Junior C. Novo método para determinação da temperatura de corpos imersos em plasma. Revista Brasileira de Aplicações de Vácuo. 2014;34(1):29-34. DOI: $10.17563 /$ rbav.v34i1.978

19. Salter TL, Bunch J, Gilmore IS. Importance of sample form and surface temperature for analysis by ambient plasma mass spectrometry (PADI). Analytical Chemistry. 2014;86(18):92649270. DOI: $10.1021 / \mathrm{ac} 502363 \mathrm{v}$

20. Amiel S, Loarer T, Pocheau C, Roche H, Gauthier E, Aumeunier $\mathrm{MH}$, et al. 2D surface temperature measurement of plasma facing components with modulated active pyrometry. The Review of Scientific Instruments. 2014;85(10):104905. DOI: $10.1063 / 1.4899210$

21. Osch EV, van der Laan JG. Material erosion and surface temperature response to plasma-disruption heat load simulations. Journal of Nuclear Materials. 1995;220-222:781-784. DOI: 10.1016/0022-3115(94)00584-2

22. Bernardelli EA, Mafra M, Maliska AM, Belmonte T, Klein AN. Influence of neutral and charged species on the plasma degradation of the stearic acid. Materials Research. 2013;16(2):385-391. DOI: $10.1590 / \mathrm{S} 1516-14392013005000008$

23. Bergman TL, Lavine AS, Incropera FP, DeWitt DP. Fundamentals of Heat and Mass Transfer. Hoboken: John Wiley and Sons; 2007.

24. Karaipekli A, Sari A, Kaygusuz K. Thermal conductivity improvement of stearic acid using expanded graphite and carbon fiber for energy storage applications. Renewable Energy. 2007;32(13):2201-2210. DOI: 10.1016/j.renene.2006.11.011

25. Matricarde Falleiro RMM, Silva LYA, Meirelles AJA, Krähenbühl MA. Vapor pressure data for fatty acids obtained using an adaptation of the DSC technique. Thermochimica Acta. 2012;547:6-12. DOI: 10.1016/j.tca.2012.07.034

26. Belmonte T, Bernardelli EA, Mafra M, Duday D, Frache G, Poncin-Epaillard F, et al. Comparison between hexatriacontane and stearic acid behaviours under late Ar? 2 post-discharge. Surface and Coatings Technology. 2011;205(Suppl 2):S443-S446. DOI: $10.1016 /$ j.surfcoat.2011.03.041

27. Tagawa M, Yokota K. Atomic oxygen-induced polymer degradation phenomena in simulated LEO space environments: How do polymers react in a complicated space environment? Acta Astronautica. 2008;62(2-3):203-211. DOI: 10.1016/j. actaastro.2006.12.043

28. Tagawa M, Minton TK. Mechanistic Studies of Atomic Oxygen Reactions with Polymers and Combined Effects with Vacuum Ultraviolet Light. MRS Bulletin. 2010;35(1):35-40. DOI: $10.1557 / \mathrm{mrs} 2010.614$

29. Praveen KM, Thomas S, Grohens Y, Mozetic M, Junkar I, Primc $\mathrm{G}$, et al. Investigations of plasma induced effects on the surface properties of lignocellulosic natural coir fibres. Applied Surface Science. 2016;368:146-156. DOI: 10.1016/j. apsusc.2016.01.159

30. Mafra M, Belmonte T, Maliska AM, da Silva Sobrinho AS, Cvelbar U, Poncin-Epaillard F. Argon-Oxygen Post-Discharge Treatment of Hexatriacontane: Heat Transfer between Gas Phase and Sample. Key Engineering Materials. 2008;373-374:421425. DOI: 10.4028/www.scientific.net/KEM.373-374.421 
31. Hody V, Belmonte T, Czerwiec T, Henrion G, Thiebaut JM. Oxygen grafting and etching of hexatriacontane in late $\mathrm{N} 2-\mathrm{O} 2$ post-discharges. Thin Solid Films. 2006;506-507:212-216. DOI: 10.1016/j.tsf.2005.08.016

32. Normand F, Granier A, Leprince P, Marec J, Shi MK, Clouet F. Polymer treatment in the flowing afterglow of an oxygen microwave discharge: Active species profile concentrations and kinetics of the functionalization. Plasma Chemistry and Plasma Processing. 1995;15(2):173-198. DOI:10.1007/ BF01459695
33. Meister J, Böhm G, Eichentopf IM, Arnold T. Simulation of the Substrate Temperature Field for Plasma Assisted Chemical Etching. Plasma Processes and Polymers. 2009;6(Suppl 1):S209--S213. DOI:10.1002/ppap.200930509

34. Zheng Z, Ren L, Feng W, Zhai Z, Wang Y. Surface characterization of polyethylene terephthalate films treated by ammonia lowtemperature plasma. Applied Surface Science. 2012;258(18):72077212. DOI: $10.1016 /$ j.apsusc.2012.04.038

35. Ma S, Bromberg V, Liu L, Egitto FD, Chiarot PR, Singler TJ. Low temperature plasma sintering of silver nanoparticles. Applied Surface Science. 2014;293:207-215. DOI: 10.1016/j.apsusc.2013.12.135 